Communications in Physics, Vol. 26, No. 4 (2016), pp. 325-334

DOI:10.15625/0868-3166/26/4/8951

\title{
STUDY OF OPTIMIZED COUPLING BASED ON MICRO-LENSED FIBERS FOR FIBERS AND PHOTONIC INTEGRATED CIRCUITS IN THE FRAMEWORK OF TELECOMMUNICATIONS AND SENSING APPLICATIONS
}

\author{
SY DAT LE ${ }^{1}$, ENGUERRAN DELCOURT ${ }^{1}$, PAULINE GIRAULT ${ }^{1}$, \\ ALDO GUTIERREZ-ARROYO ${ }^{1}$, PAUL AZUELOS ${ }^{1}$, NATHALIE LORRAIN ${ }^{1}$, \\ LOÏC BODIOU $^{1}$, LUIZ POFFO ${ }^{1}$, JEAN-MARC GOUJON ${ }^{1}$, YANNICK DUMEIGE ${ }^{1}$, \\ ISABELLE HARDY $^{1}$, PHILIPPE ROCHARD ${ }^{1}$, JONATHAN LEMAITRE ${ }^{1}$, \\ PARASTESH PIRASTEH ${ }^{1}$, MOHAMMED GUENDOUZ ${ }^{1}$, THIERRY CHARTIER ${ }^{1}$, \\ LIONEL QUÉTEL ${ }^{2}$, SÉBASTIEN CLAUDOT ${ }^{3}$, \\ JOËL CHARRIER ${ }^{1}$ AND MONIQUE THUAL ${ }^{1, \dagger}$ \\ ${ }^{1}$ Foton UMR CNRS 6082, Université de Rennes 1, 22305, CS80518 Enssat Lannion \\ ${ }^{2}$ IDIL Fibres optiques, 21 rue Louis De Broglie 22300 Lannion, France \\ ${ }^{3}$ Souriau - Esterline ECT, RD323, 72470 Champagné, France \\ ${ }^{\dagger}$ E-mail: monique.thual@univ-rennes1.fr \\ Received 01 December 2016 \\ Accepted for publication 16 January 2017
}

\begin{abstract}
We demonstrate the interest of expanded beam microlenses (around $55 \mu$ m of mode field diameter) to relax positioning tolerances and to decrease reflectance in single mode fiber to fiber interconnections. We also point out the interest of micro-lenses of very small mode field diameter (around $2 \mu \mathrm{m}$ ) to improve coupling efficiency in specialty fibers and integrated waveguides for non linear effects based functions and for sensors applications at a wavelength of $1.55 \mu \mathrm{m}$.
\end{abstract}

Keywords: optical interconnection, fiber optics, integrated optics, microlenses.

Classification numbers: 42.81.Pa, 84.40. Ua. 


\section{INTRODUCTION}

Injecting optical signal from a single mode fiber (SMF) to a fiber or an integrated waveguide is a common problem encountered in many kinds of applications. In the field of optical telecommunications, laser to fiber, fiber to fiber, or fiber to integrated optics connections is a topic that has led to many scientific theoretical and technological works since 1964 [1-10]. For that purpose microlenses have been developed whose main role is to match the mode field diameters (MFD) of the fibers and waveguides to be coupled in order to improve coupling efficiency $[5,6]$. In other cases the role of the microlenses is to expand the MFD in order to relax lateral and axial positioning tolerances, to decrease the sensitivity to high power and for harsh environments fiber to fiber connections [10]. A lot of solutions are based on a graded index section spliced at the fiber output $[11,12]$. This subject has continued to generate great interest up to nowadays in particular with the deployment of fiber to the home networks [11]. Moreover, the interest in optical coupling solutions remains very strong since the arrival of photonics in many industrial sectors such as automotive, avionics, mechanical engineering, biology and health.

After a brief presentation of their principle, we point out the interest of micro-lenses based on graded index sections spliced at the fiber output we have developed to improve coupling light in devices. Some examples of coupling in different kinds of applications are given in the Infra Red wavelength range.

\section{COUPLING LIGHT}

\section{Mode adaptation for coupling improvement}

Coupling efficiency between a SMF and a waveguide whose transverse intensity distributions are gaussian depends on the mismatch between their MFD. As the coupling efficiency is the overlap integral between the two field amplitudes, it is the higher when the two fields are the same and decreases with their mismatch. Moreover, the larger the MFD, the higher the lateral and axial positioning tolerances, and the smaller the angular ones.

In order to improve coupling efficiency, we have developed microlenses at fiber outputs whose MFD can be varied as a function of the application. The interest is that we are able to adapt it to the coupling of MFD as low as $2 \mu \mathrm{m}$ up to $55 \mu \mathrm{m}$ to fulfill the requirements of coupling efficiency improvement for numerous applications.

\section{a. Expanded beam microlenses}

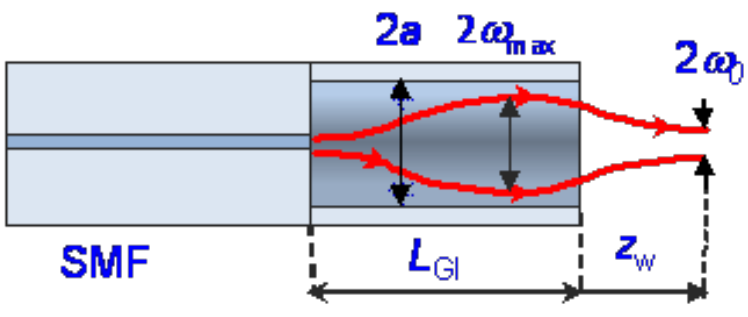

Fig. 1. Principle diagram of the SMF-GIF microlens. 
The microlenses we have developed consist of a parabolic transverse profile graded index fiber (GIF) spliced to a SMF as shown in Fig. 1. Thanks to the graded index profile, the MFD $(2 \omega)$ varies periodically in the GIF core whose diameter is $2 \mathrm{a}$. We cut the GIF at the appropriate distance $L_{G I F}$ from the splice to obtain a SMF-GIF microlens whose MFD ( $\left.2 \omega_{0}\right)$ and working distance $\mathrm{z}_{w}$, also depending on the core diameter $2 \mathrm{a}$ and the GIF profile, are appropriate with the application at a given wavelength.

For expanded beam applications we cut the GIF at the distance $L_{G I F}$ where the MFD is maximum $\left(2 \omega_{\max }\right)$. The theoretical concepts, coupling efficiency, and reflectance analysis we have developed for that purpose are detailed in [13].

\section{b. Small beam microlenses}

In order to achieve very small MFD we add a silica droplet at the GIF output where the MFD is maximum. The microlens end profile shape is hyperbolic (HYP) to avoid spherical aberrations [12]. The MFD $\left(2 \omega_{0}\right)$ and working distance $\left(z_{w}\right)$ of the SMF-GIF-HYP microlens depend on the GIF characteristics and length as well as on the radius of the osculating sphere of the hyperbolic profile as shown in Fig. 2.

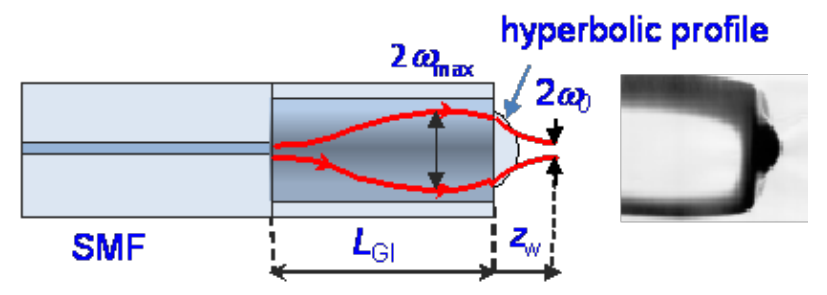

Fig. 2. Principle diagram of the SMF-GIF-HYP microlens.

\section{APPLICATIONS}

Expanded beam microlenses that we have developed have shown their interest for relaxing sensitivity to positioning tolerances, whereas very small mode field diameter micro-lenses are very useful to improve coupling efficiency to specialty fibers or waveguides for non-linear based effects devices or for sensor applications as will be shown.

\section{III.1. Fiber to fiber coupling applications}

\section{a. Expanded beam connection}

We have designed and fabricated SMF-GIF microlenses [11] whose MFD $2 \omega_{0}$ is extended to $55 \mu \mathrm{m}$ for relaxing tolerances sensitivity to lateral and axial positioning and to harsh environments fiber to fiber connections compared with SMF as shown in Table 1.

Table 1. Positioning tolerances for $1 \mathrm{~dB}$ excess losses.

\begin{tabular}{|l|l|l|l|l|}
\hline Connection & $2 \omega_{0}(\mu \mathrm{m})$ & $\Theta\left(^{\circ}\right)$ & $\Delta \mathrm{x}(\mu \mathrm{m})$ & $\Delta \mathrm{z}(\mu \mathrm{m})$ \\
\hline SMF & 10.5 & 2.6 & 2.5 & 57 \\
SMF GIF & 55 & 0.5 & 13 & 1560 \\
\hline
\end{tabular}


We have demonstrated the interest of those SMF-GIF microlenses for low-loss physical contact LC connections as seen in Fig. 3, with coupling losses as low as $0.5 \mathrm{~dB}$ in $\mathrm{O}$ and $\mathrm{C}$ Band, without penalty compared with SMF in a $50 \mathrm{~km} 10 \mathrm{Gbit} / \mathrm{s}$ transmission (Fig. 4). Moreover the return loss is very interesting $(62 \pm 2 \mathrm{~dB})$ compared with SMF Physical Contact ( $54 \pm 2 \mathrm{~dB})$.

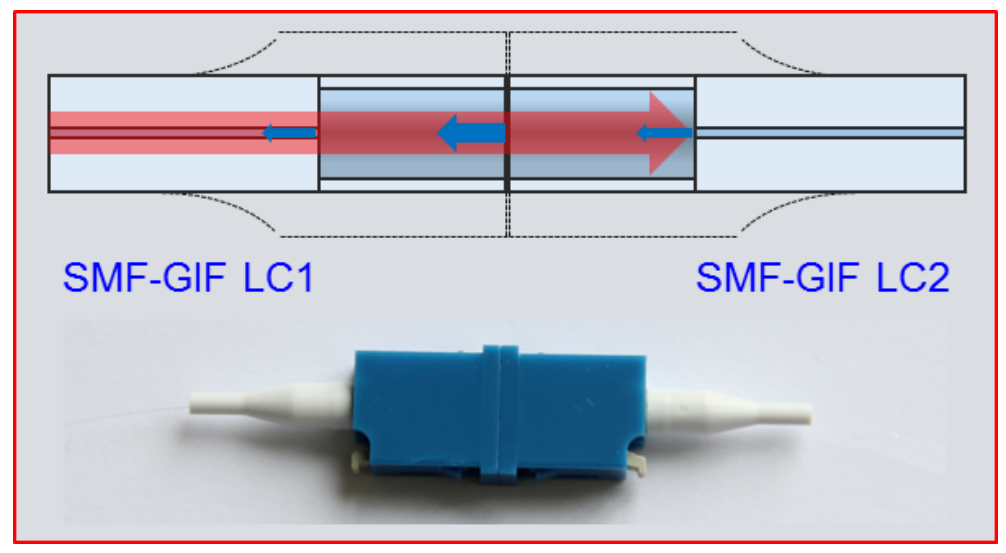

Fig. 3. SMF-GIF in LC connection.

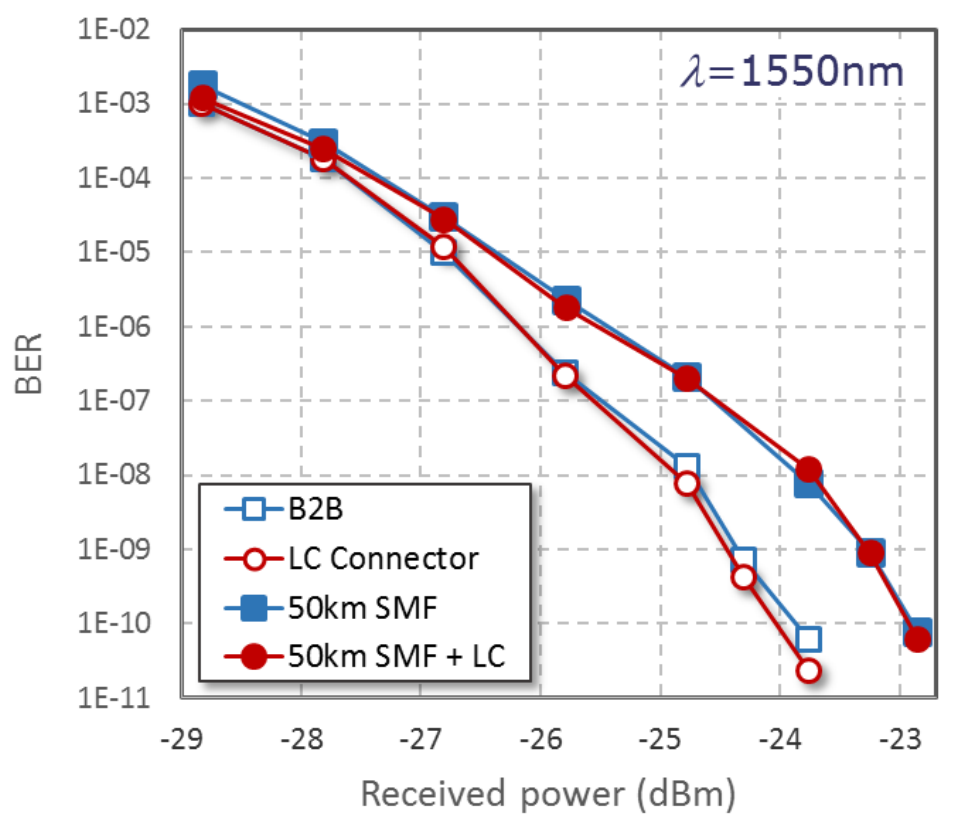

Fig. 4. Bit Error Rate of SMF GIF LC connections compared to both Back to Back and $50 \mathrm{~km}$ SMF $10 \mathrm{Git} / \mathrm{s}$ transmission. 


\section{b. Highly-nonlinear fibers for telecommunication applications}

Microstructured chalcogenide fibers are of great interest in the field of all-optical signal processing because of their high nonlinearity (four orders of magnitude higher than standard fibers). Using a microstructured chalcogenide fiber fabricated at the University of Rennes 1 [14], we have experimentally demonstrated all-optical demultiplexing of a $170.8 \mathrm{Gbit} / \mathrm{s}$ on-off keying signal in a chalcogenide GeAsSe microstructured fiber [15]. The structure of the fiber is designed with three rings of holes around a solid core as seen in Fig. 5. The fiber is fabricated with a core diameter as small as possible to enhance the nonlinear coefficient $\left(\gamma=25000 \mathrm{~W}^{-1} \mathrm{~km}^{-1}\right)$ while the core diameter of the input and output ends is kept larger to reduce the coupling loss. The geometrical parameters of the fiber are summarised in Fig. 5. SMF-GIF-HYP micro-lensed fibers with a mode field diameter of $3.2 \mu \mathrm{m}$ and a working distance of $30 \mu \mathrm{m}$ were used for coupling optimization at both ends of the fiber.

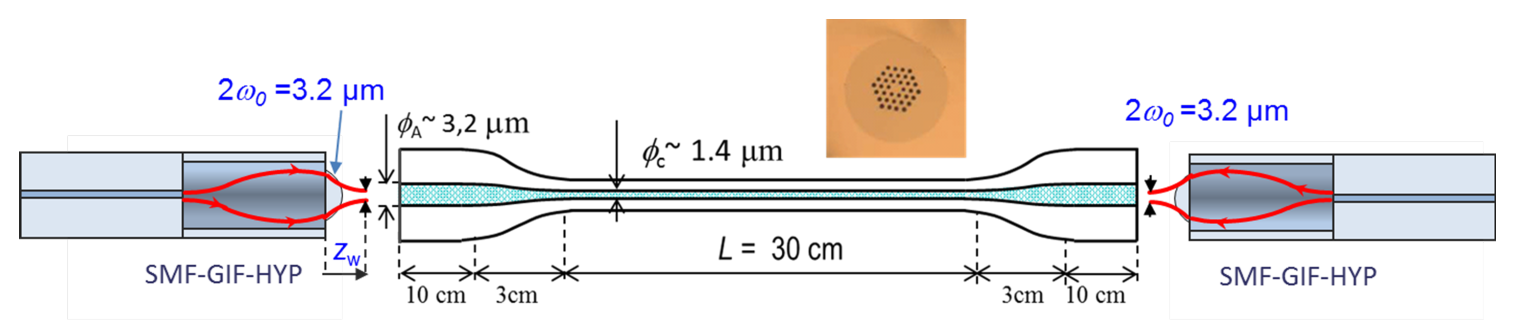

Fig. 5. Chalcogenide fiber and its micro-lensed fibers for coupling optimization.

To demonstrate all-optical demultiplexing, a $170.8 \mathrm{Gbit} / \mathrm{s}$ on-off keying signal is injected together with a clock signal at $42.7 \mathrm{GHz}$ in the chalcogenide fiber as depicted in Fig. 6(a). The $170.8 \mathrm{Gbit} / \mathrm{s}$ signal is generated by time-multiplexing four $42.7 \mathrm{Gbit} / \mathrm{s}$ signals. Figs. 6(b) and 6(c) show the spectra at the input and at the output of the GeAsSe fiber, respectively. Due to efficient four-wave mixing (FWM) in the fiber, an idler wave, down-shifted with respect to the clock wavelength and corresponding to one tributary channel at $42.7 \mathrm{Gbit} / \mathrm{s}$, is generated. A maximum FWM idler power at the output of the chalcogenide fiber is found when both input signals are well synchronised. By varying the optical delay line by a step of one bit time (5.9 ps in our case), one can choose which $42.7 \mathrm{Gbit} / \mathrm{s}$ tributary channel is demultiplexed from the $170.8 \mathrm{Gbit} / \mathrm{s}$ signal. The total average power is $56 \mathrm{~mW}$ at the output of the microlensed injection fiber, including a $42.7 \mathrm{GHz}$ clock power of $32 \mathrm{~mW}$. At the output of the GeAsSe fiber, a $1 \mathrm{~nm}$ filter is used to extract the idler wave centred at $1545.4 \mathrm{~nm}$. Fig. 6(d) shows an example of the eye diagram of one demultiplexed $42.7 \mathrm{Gbit} / \mathrm{s}$ tributary channel.

\section{III.2. Fiber to waveguide coupling applications}

Another application is to obtain coupling efficiency between a SMF and a photonic integrated circuit. Indeed, photonic packaging is still on the fiber optics platform: fiber-pigtailed, discrete devices or small-scale combinations of integrated devices. The microlenses are used to inject optical signal from a SMF to integrated waveguides based on different materials for non linear or sensors optical applications. 

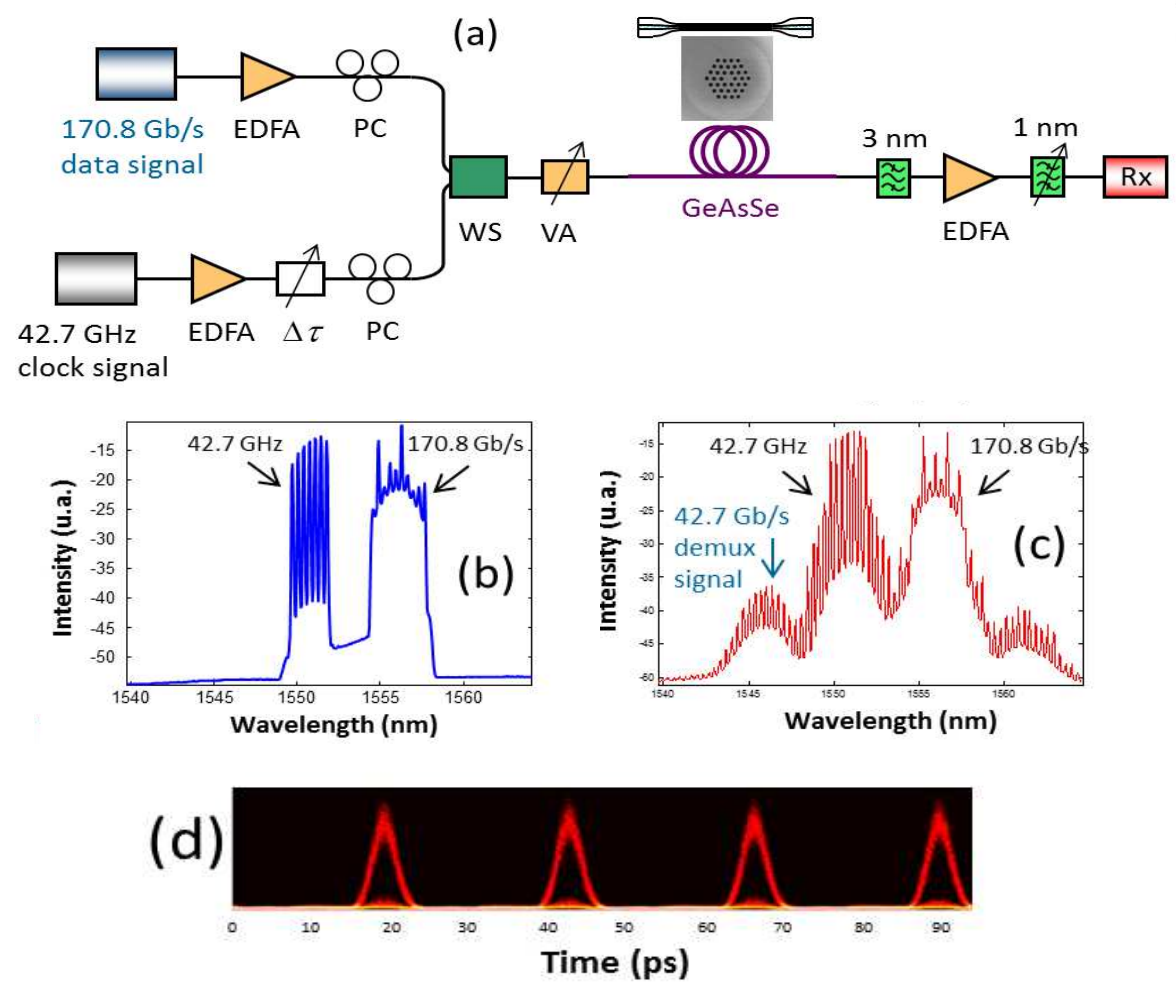

Fig. 6. Setup (a) and results on all-optical demultiplexing in a chalcogenide fiber (b), (c) and (d).

\section{a. Chalcogenide integrated waveguides for non linear applications}

Third-order nonlinear optical phenomena have been intensely studied in integrated devices [16-19] for different applications. The third order nonlinear efficiency of such devices can be improved by increasing the waveguide nonlinear parameter $\gamma$, which can be obtained by reducing effective area or using a high nonlinear glass $\left(\mathrm{n}_{2}\right)$. Integrated optical waveguides were fabricated based on chalcogenide films. Indeed, besides their wide transparency window (1-20 $\mu \mathrm{m})$, chalcogenide films offer both a strong nonlinearity ( $\mathrm{n}_{2}$ values up to $\sim 700$ times that of silica) and low nonlinear absorption [20].

This chalcogenide platform could therefore allow the development of efficient, low-power operation optical devices for telecommunications and on-chip wavelength division multiplexing (WDM) optical interconnects.

Ridge waveguides of geometrical dimensions, for guiding layer, $600 \mathrm{~nm} \times 400 \mathrm{~nm}$, were fabricated using a classical i-line photolithographic process and optical characterizations were conducted by using microlenses to couple efficiency the light to the waveguide (Fig. 7 (a)). Strong nonlinear spectral broadening due to Self Phase Modulation (SPM) was measured around 1.55 $\mu \mathrm{m}$ by using $9.6 \mathrm{ps}$ optical pulses and FWM in cm-long single-mode waveguides by using $\mathrm{CW}$ laser pump at $1.55 \mu \mathrm{m}$ were also observed. Figs. 7(b) and 7(c) are some examples of obtained experimental results. 

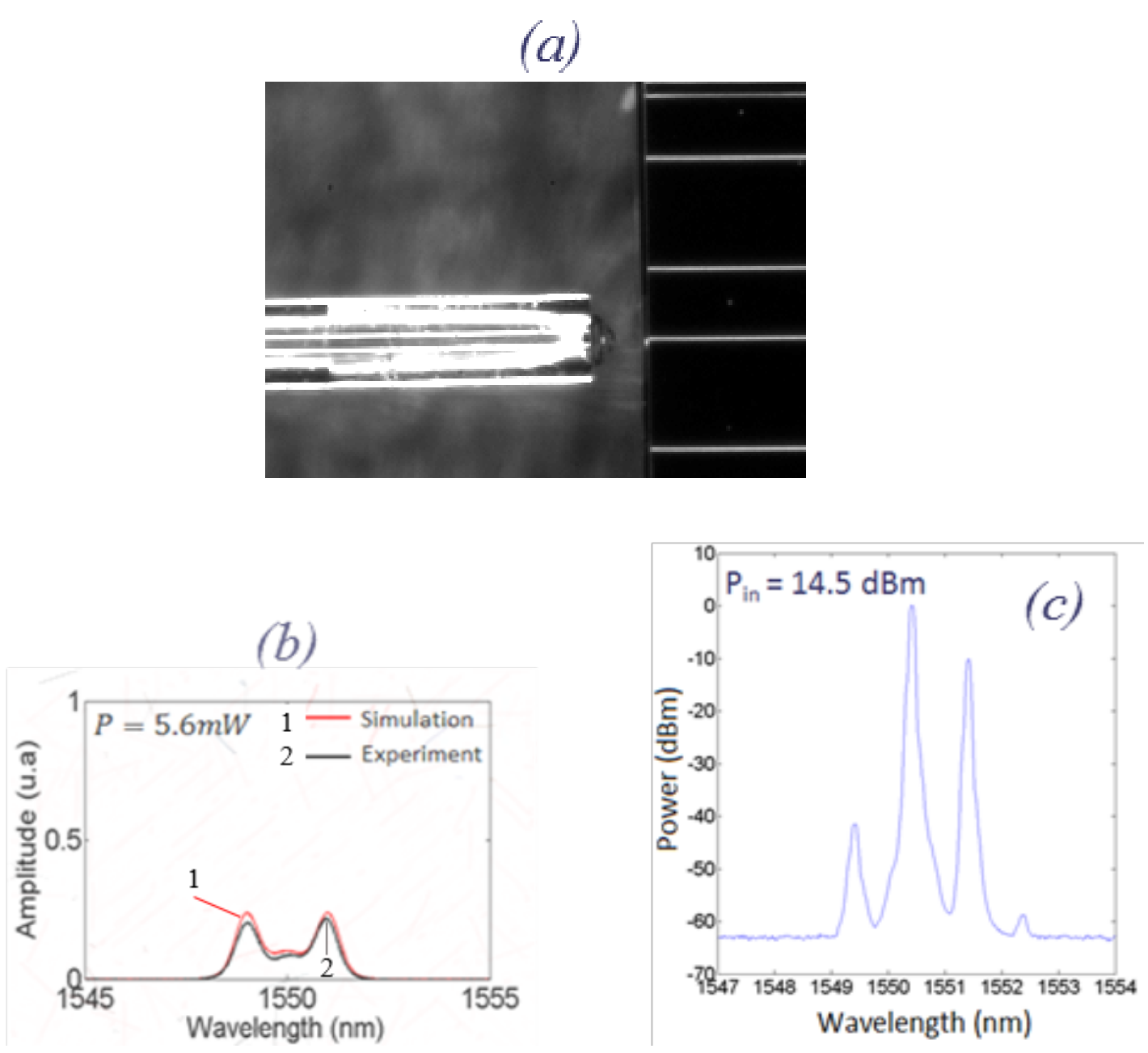

Fig. 7. (a) micro-lensed fiber and integrated waveguide photograph for coupling optimization; (b) Experimental spectrum measured at the waveguide output for $5.6 \mathrm{~mW}$ power to observe the SPM; (c) Experimental spectrum for a $1.1 \mathrm{~cm}$ long ChGs ridge waveguide to measure FWM.

\section{b. Microring resonators for sensor applications}

The microlensed fibers were also used for the optical characterizations of a racetrack integrated micro-resonator (MR), made of porous silica (PS) ridge waveguides [21], and destined to sensing applications. In this context, the aim of this work is to improve sensitivities of sensors based on easily integrated MR components by the use of a porous material. The MR structure is based on PS ridge waveguides. The dimensions of the porous silica core waveguide $(1.8 \times 2.5$ $\mu \mathrm{m}^{2}$ ), have been chosen to provide single mode propagation at $1.55 \mu \mathrm{m}$. The MR, made by using standard photolithography process, has been well achieved with a separation distance between the straight waveguide and the racetrack waveguide of $0.8 \pm 0.1 \mu \mathrm{m}$. 


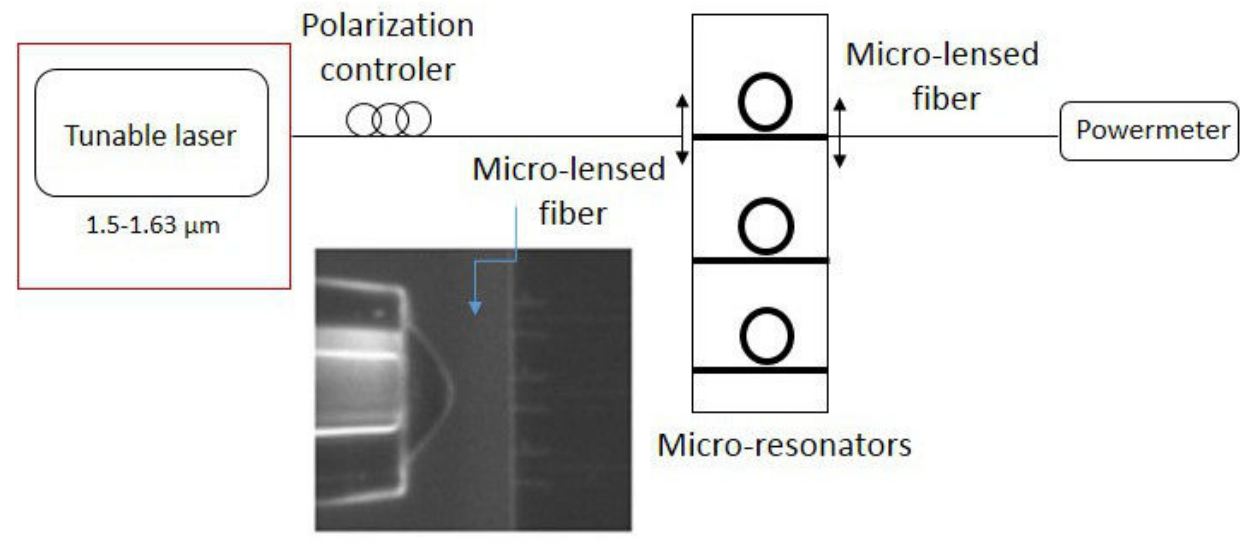

(a)

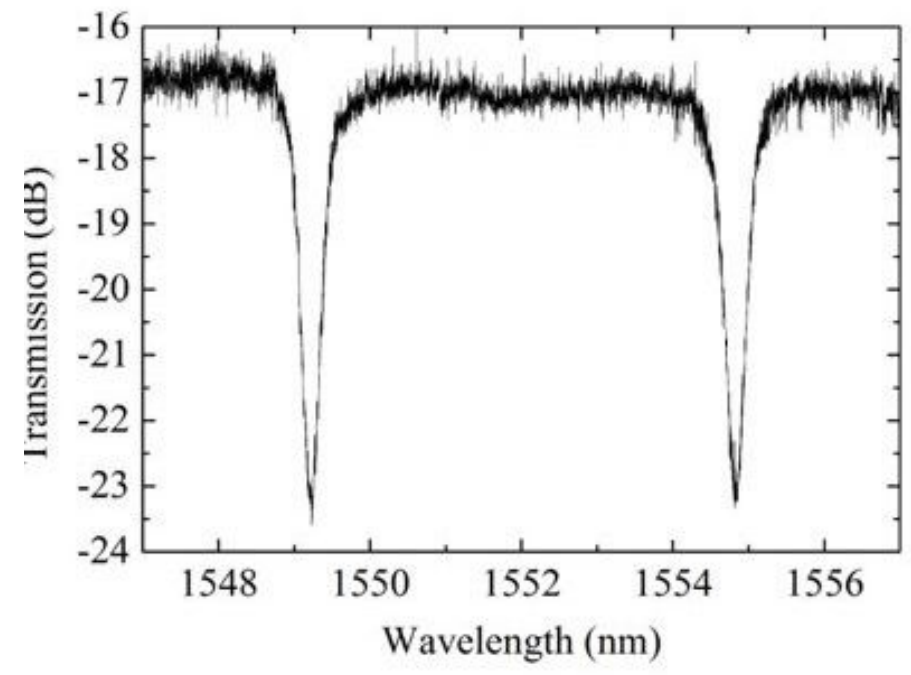

(b)

Fig. 8. (a) Schema of the optical bench with 2 micro-lensed fibers (b) Experimental transmission spectra for one polarization of light.

In order to verify that the porous silica racetrack MR is optically operational, the spectral transmission response of the MR was measured. The output from a tunable wavelength laser is injected in the input straight waveguide using a micro-lensed fiber with a MFD of $2.2 \mu \mathrm{m}$ and constant polarization. A second micro-lensed single mode ber is also used to couple the output signal of the straight waveguide to a powermeter (Fig. 8(a)). The measured transmission as a function of the wavelength for one polarization of light is reported in Fig. 8(b). It demonstrates that the light is propagated in the waveguide and the coupling of the light occurs between the access waveguide and the MR. 


\section{CONCLUSION}

First, we have demonstrated the interest of expanded beam connectors based on microlenses of 55- $\mu \mathrm{m}$ MFD to relax positioning tolerances in a low loss $(0.5 \mathrm{~dB})$ and high return loss $(62$ $\mathrm{dB})$ fiber to fiber interconnection in an error free transmission at a bit rate of $10 \mathrm{Gbit} / \mathrm{s}$. Then, we have pointed out the use of microlenses of very small mode field diameters (around $2 \mu \mathrm{m}$ ) for injecting signal in specialty chalcogenide micro-structured fibers and integrated waveguides to increase third order non-linear effects as SPM and FWM for optical communication functions such as demultiplexing. The same kinds of microlenses with very small mode field diameters have also been used to characterize porous MR based sensors sensitive to refractive index variation. These microlenses allow to improve the coupling efficiency into the access integrated waveguide of the MR and at its output towards a spectrum analyser. Another application would consist in developing the coupling solutions in the Mid-Infrared wavelength range. Indeed, photonic integrated circuits and the light coupling between fibers and fiber/chip are a quasi-unexplored research field, but with a huge potential for environmental, biomedical and security monitoring and detection applications.

The impact of this new technology cannot be underestimated. This also will open up a new level of research.

\section{ACKNOWLEDGMENTS}

The present research was supported by the French government R\&D funding, the regional council of Brittany and the Lannion Tregor Community.

\section{REFERENCES}

[1] H. Kogelnik in Proceeding Symposium Quasi-Optics, Vol. 14 (1964) 333-347.

[2] M. Saruwatari and K. Nawata, Applied Optics Vol. 18 (1979) 1847-1856.

[3] A. Nicia, Applied Optics, Vol. 20 (1981) 3136-3145.

[4] P. Chanclou, M. Thual, J. Lostec, P. Auvray, J. Caulet, G. Joulié, A. Poudoulec, B. Clavel, Electronic Letters Vol. 34 (1998) 273-274.

[5] K. Shiraishi, Shin-Ichi Kuoo, Journal of Lightwave Technology, Vol. 18 (2000) 787-794.

[6] M. Thual, P. Chanclou, O. Gautreau, L. Caledec, C. Guignard and P. Besnard, Electronic Letters, 39 (2003) 1504-1505

[7] R. Bachelot, A. Fares, R. Fikri, D. Barchiesi, G. Lerondel, P. Royer, Optics letters, Vol. 29 (2004) 1-3.

[8] D. S. Kokkinos, C. Saravanos, W. Stanford, W. Wang, and Y. Hua, “Optical Fiber Communication Conf. (OFC), Anaheim, C.A., US, 2006, paper NTuA5.

[9] M. Thual. D. Malardé, B. Abhervé-Gueguen, P. Rochard, P. Chanclou, Optical Engineering 46 (2007) 1-12.

[10] D. Childers, M. Hughes, S. Lutz, and T. Satake, Optical Fiber Communication Conf. Los Angeles, C.A., US 2015, paper ThC1, Invited paper, 2015

[11] S. D. Le, M. Gadonna, M. Thual, L. Quetel, J.-F. Riboulet, V. Metzger, D.s Parker, A. Philippe, and S. Claudot, oral session, section D1.5 W4B.5, Los Angeles, Optical Fiber Communications 2015.

[12] M. Thual, G. Moreau, J. Ribette, P. Rochard, M. Gadonna, J. C. Simon, Optics Communications, Vol. 255 (2005) 278-285.

[13] S. D. Le, P. Rochard, J.-B. Briand, L. Quétel, S. Claudot, and M. Thual, Journal of Ligthwave Technology, 34 (2016) 2029-2099.

[14] P. Toupin, L. Brilland, J. Troles, J.-L. Adam, Opt. Mater. Express, Vol. 2 (2012) 1359-1366.

[15] S. D. Le, M. Gay, L. Bramerie, T. Chartier, M. Thual, J.-C. Simon, L. Brilland, D. Méchin, P. Toupin and J. Troles, Electron. Lett., Vol. 49 (2012) 136-138.

[16] A. C. Turner, M. A. Foster, A. L. Gaeta, and M. Lipson, Optics Express 16 (2008) 4881-4887. 
[17] F. Luan, M. D. Pelusi, M. R. Lamont, D.-Y. Choi, S. Madden, B. Luther-Davies, and B. J. Eggleton, Optics Express 17 (2009) 3514-3520.

[18] S. Azzini, D. Grassani, M. Galli, L.C. Andreani, M. Sorel, M.J. Strain, L.G. Helt, J.E. Sipe, M. Liscidini, and D. Bajoni, Optics Letters 37 (2012) 3807-3809.

[19] D.J. Moss, R. Morandotti, A.L. Gaeta, and M. Lipson, Nature Photonics 7 (2013) 597-607.

[20] M. Olivier, J. Tchahame, P. Nemec, M. Chauvet, V. Besse, C. Cassagne, G. Boudebs, G. Renversez, R. Boidin, E. Baudet, and V. Nazabal, Optical Materials Express 4 (2014) 525-540.

[21] P. Girault, N. Lorrain, J. Lemaitre, L. Poffo, M. Guendouz, I. Hardy, M. Gadonna, A. Gutierrez, L. Bodiou and J. Charrier. Optical Materials, 50 (2015) 167-174. 